\title{
Functional food ingredients for control of gestational diabetes mellitus: a review
}

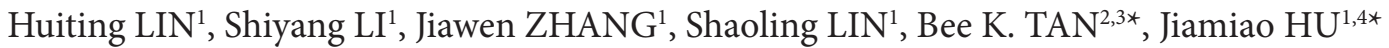

\begin{abstract}
Gestational diabetes mellitus (GDM) is highly prevalent worldwide, with an estimated 10-15\% percent of pregnancies affected. Increasing evidence indicates that functional food ingredients (FFIs) may help relieve GDM via multiple mechanisms, such as eliminating free radicals, downregulating inflammation, promoting insulin secretion and signaling, targeting hepatic gluconeogenesis and glycogen storage metabolism, and regulating the intestinal microflora. In this article, the effects of functional food ingredients on GDM and the possible underlying mechanisms of action are reviewed. This review provides reference information that can be useful for the development of novel functional supplements for the control of GDM.
\end{abstract}

Keywords: gestational diabetes mellitus; food ingredients; insulin signaling; oxidative stress; intestinal microflora.

Practical Application: Used as natural substances for GDM management.

\section{Introduction}

Gestational diabetes mellitus (GDM) refers to carbohydrate intolerance resulting in hyperglycemia that is first diagnosed during pregnancy. It is characterized by high blood glucose levels in pregnant women who have not been previously diagnosed with diabetes (Patti et al., 2018). The cause of GDM is multifactorial (Dean et al., 2014); age, pre-gestational obesity and a family history of diabetes have been identified as risk factors for GDM (Cho et al., 2016). GDM was thought to be a transient state and that glucose homeostasis would recover shortly after delivery (Ponzo et al., 2019). However, increasing evidence demonstrates that GDM has profound and long-lasting adverse effects on both the mother and child. Without proper treatment, GDM may increase perinatal morbidity and mortality (Farrar et al., 2017). Moreover, long-term metabolic risks are also being recognized in women who had GDM as well as in their offspring (Stewart, 2020). GDM pregnancies may increase the risk of type 2 diabetes in the mother by $30-50 \%$ within 10 20 years, while the offspring show an increased risk of childhood obesity and diabetes mellitus (Johns et al., 2018). Currently, diet intervention is recommended for women with GDM, especially those with mild GDM, in addition to drug interventions i.e. metformin, insulin, with its potential side effects. In addition to general guidelines, such as switching to low glycemic index foods and/or reducing carbohydrate intake, a variety of functional food may help control this condition or make GDM less likely to occur. Functional foods have been defined as industrially processed or natural foods that, besides providing basic nutrition, beneficially promote optimal health and help reduce the risk of disease when consumed on a regular basis (Granato et al., 2020). Increasing number of non-conventional food plants show great potential to be novel functional foods (Folharini et al., 2019). Particularly, functional foods are often rich in bioactive compounds associated with prevention of metabolic disease (Konstantinidi \& Koutelidakis, 2019). Indeed, the beneficial effects of various functional food ingredients (FFIs) on GDM have been demonstrated using experimental animal models as well as human trials. However, the mechanisms of action underlying the beneficial effects of FFIs on GDM are not fully understood. We will focus on these mechanisms in this review.

\section{Experimental animal models to evaluate the effects of FFIs on GDM}

Although food ingredients are usually safe, safety concerns cannot be overlooked. Therefore, despite invaluable clinical data on the beneficial effects of FFIs on GDM in humans, animal models are also widely used in GDM studies. In general, rodent models of GDM can be divided into the following three types: 1) genetically engineered rodent models of GDM. For instance, $\mathrm{C} 57 \mathrm{BL} / \mathrm{Ks})^{\mathrm{db} /+}(\mathrm{db} /+)$ mice, a typical diabetes model, harbor a heterozygous mutation in the leptin receptor gene Lepr and can mimic gestational diabetes. In general, six- to eight-week-old mice are used; 2) Alloxan/Streptozotocin (STZ)-induced GDM models. Chemical agents alloxan and STZ selectively damage pancreatic $\beta$-cells, resulting in hyperglycemia and diabetes with its attending complications (Pasek \& Gannon, 2013). Therefore, alloxan/STZ administration during/before pregnancy can be used to mimic both severe and mild GDM, depending on the dose (Kiss et al., 2009). This treatment is generally supplemented with high-fat high-fructose (HFHF) fodder. 3) Obesogenic diet-induced GDM models. Pre-pregnancy overweight and excess gestational weight gain have been identified as important causes of GDM. Therefore, obesogenic diets, such as high-fat high-fructose diets, or cafeteria-style foods are also widely used 
to mimic GDM. ICR mice, Wistar rats, and Sprague Dawley rats are usually used for this purpose. Admittedly, the causes of GDM are complex; therefore, the previous rodent models are unlikely to fully mirror the pathogenesis of GDM in humans. Choosing a suitable rodent model is crucial for evaluating FFIs before human trials. Continued development of animal models is essential to investigate FFIs as potential treatments or preventive therapies for GDM.

\section{Molecular mechanisms}

\subsection{FFIs increase insulin secretion and potentiate insulin signaling}

Insulin signaling is believed to play a central role in the regulation of glucose homeostasis. Lack of insulin secretion and insulin sensitivity results in hyperglycemia in GDM patients. Not surprisingly, various FFIs with anti-GDM properties have shown positive effects on insulin secretion and the insulin signaling pathway. For example, Li et al. (2019) found that naringenin showed great potential for treatment of GDM due to its insulin sensitivity-enhancing effect in skeletal muscle; this is dependent on AMPK activation. Zou et al. (2018) reported that mogroside IIIE greatly improved insulin sensitivity by activating the AMPK/HDAC4/G6Pase signaling pathway, alleviating GDM in mice. Plows et al. (2020) reported that intracellular insulin signaling in adipose tissue could be improved by adding myoinositol and probiotics, although neither myoinositol nor probiotics improved glucose tolerance. PPARs are wellcharacterized transcriptional regulators with key functions in insulin metabolism (Wahli \& Michalik, 2012). Indeed, PPAR agonists have a long history as effective treatments for type 2 diabetes (T2D). Therefore, FFIs exhibiting PPAR-activating properties (often weak PPAR agonists) could be explored as anti-GDM agents. For instance, Sun et al. (2019) showed that pomegranate ellagic polyphenols activated PPARa-TRB3-AKT2p-FOXO1-GLUT2 signaling (associated with insulin sensitivity) in a dose-dependent manner. Fornes et al. (2020) reported that a diet supplemented with $6 \%$ olive oil prevented the increase in PPAR $\gamma$ and PPAR $\delta$ levels in the male fetuses of pregnant rats. On the other hand, Capobianco reported that a maternal diet supplemented with polyunsaturated fatty acids attenuated mTOR signaling, preventing fetal overgrowth (Capobianco et al., 2018b). These observations indicate a possible relationship between improved insulin sensitivity and the anti-GDM effects of FFIs.

\subsection{FFIs target hepatic gluconeogenesis and glycogen storage}

GDM is associated with inhibition of glycogen synthesis, affecting the citric acid cycle and gluconeogenesis (Gao et al., 2019b). One study reported that the prevalence of non-alcoholic fatty liver disease among women with a history of GDM was 14-38\% higher than in women without such history (Sattari et al., 2020). Moreover, hepatic insulin resistance can result in increased release of glucose (from gluconeogenesis) and free fatty acids (due to enhanced lipolysis), leading to fetal overgrowth (Lee et al., 2019). Several studies have revealed that normalization of glycogen synthesis in the liver is one mechanism by which FFIs relieve GDM. For instance, Zhang et al. (2020) reported that astragaloside IV treatment reduced hepatic gluconeogenesis by downregulating cAMP accumulation in the liver of GDM mice. Yao et al. (2015) reported that resveratrol influenced glucose production in the fetus by reducing the enzymatic activity of G6P, and that the AMPK signaling pathway was the potential target of resveratrol in GDM. Furthermore, Brawerman et al. (2019) showed that hepatic glucose metabolism in the offspring of GDM rats could be restored by maternal resveratrol administration and that the utilization of glucose was enhanced by glycolytic flux. These studies support the notion that FFIs can modulate cellular and whole-body energy homeostasis under stress conditions during pregnancy by activating the energy sensor system: AMPK (Kumagai et al., 2018). Besides, SREBP-1 is also considered a target to improve hepatic glycogen content and relieve GDM. Hua et al. (2016) found that diosgenin supplementation normalized gluconeogenesis by increasing expression of Srebp-1 and its target genes, attenuating FAS, SDC-1, ACC. In conclusion, hepatic gluconeogenesis and glycogen storage are promising FFI targets for the treatment of GDM.

\subsection{FFIs ameliorate GDM-induced oxidative stress}

GDM is a pathological condition often associated with oxidative stress (Zygula et al., 2019). Reduced activity of endogenous antioxidant enzymes such as CAT, SOD, TBARS, GPX, and GSH is a common finding in GDM pregnancies. As shown in Table 1, administering FFIs to GDM mice significantly enhances the activity of antioxidant enzymes and attenuates GDM outcomes. For example, Sha et al. (2019) reported that mangiferin attenuated GDM outcomes by increasing GPX, GSH, SOD, and CAT levels in the placenta of GDM mice. Likewise, Hua et al. (2016) determined that diosgenin could ameliorate oxidative stress in pregnant $\mathrm{db} /+$ mice, as evidenced by decreased TBARS, and upregulation of GSH levels and SOD and CAT activity. Similar conclusions were reported in a study by Hosni et al. which showed that the defective antioxidant defense system in GDM could be improved by daily supplementation with $20 \mathrm{mg} / \mathrm{kg}$ cinnamaldehyde (Hosni et al., 2017). Therefore, one of the mechanisms by which FFIs exert beneficial effects in pregnancies complicated by GDM appears to be by inhibiting oxidative stress.

\subsection{FFIs attenuate GDM-induced inflammation}

GDM is often strongly associated with an inflammatory state, as evidenced by increased levels of proinflammatory cytokines like IL- $\beta$, IL-6, and TNF- $\alpha$, and elevated CRP (Pantham et al., 2015). Suppression of inflammatory responses is also believed to be a mechanism by which FFIs relieve the deleterious outcome of GDM. For example, treatment of GDM mice one week before mating with cinnamaldehyde had glucose lowering effects and reduced the levels of proinflammatory cytokines like TNF- $\alpha$, as well as MDA and NO (Hosni et al., 2017). Another study suggested that apocynin (4-hydroxy-3-methoxyacetophenone) reduced the serum levels of glucose by downregulating IL- $1 \beta$, IL- 6 , and TNF- $\alpha$, while activating the TLR4/NF- $\kappa B$ signaling pathway (Liu et al., 2020). Zou et al. (2018) reported that a maternal diet supplemented with mogroside IIIE reduced pancreatic and serum levels of IL- $1 \beta$, IL- 6 , and TNF- $\alpha$, while enhancing AMPK activity and reducing G6Pase production. Nguyen-Ngo et al. (2020) also 
Table 1. Effects of FFIs on molecular mechanisms associated with GDM.

\begin{tabular}{|c|c|c|c|}
\hline Model & Intervention & Mechanism & Reference \\
\hline $\mathrm{C} 57 \mathrm{BL} / \mathrm{Ks})^{\mathrm{db} /+}$ mice & Resveratrol at $10 \mathrm{mg} / \mathrm{kg}^{\star} \mathrm{d}$ & $\begin{array}{c}\text { body weight } \downarrow \text {; glucose } \downarrow \text {; insulin sensitivity } \uparrow ; \\
\text { G6P } \downarrow \text {; AMPK signaling } \uparrow \text {; p-AMPK/AMPK } \uparrow ; \\
\text { p-HDAC4/HDAC4 } \downarrow \text {; }\end{array}$ & Yao et al. (2015) \\
\hline $\mathrm{C} 57 \mathrm{BL} / \mathrm{Ks} J^{\mathrm{db} /+}$ mice & Naringenin at $100 \mathrm{mg} / \mathrm{kg}{ }^{*} \mathrm{~d}$ & 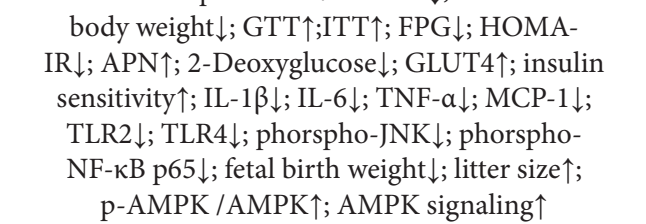 & Li et al. (2019) \\
\hline $\begin{array}{l}\text { C57BL/KsJ- } \\
\text { Lep }^{\mathrm{db} /+} \text { mice }\end{array}$ & Naringenin at $50 \mathrm{mg} / \mathrm{kg}^{\star} \mathrm{d}$ & $\begin{array}{l}\text { SOD } \uparrow ; \text { GSH } \uparrow ; \text { MDA } \downarrow \text {; body weight } \uparrow \text {; glucose } \downarrow \text {; } \\
\text { insulin } \uparrow \text {; offspring(litter size } \uparrow \text {;itter weight } \downarrow \text { ) }\end{array}$ & Xing et al. (2016) \\
\hline $\mathrm{C} 57 \mathrm{BL} / \mathrm{Ks} \mathrm{J}^{\mathrm{db} /+}$ mice & Mangiferin at $50 \mathrm{mg} / \mathrm{kg}^{*} \mathrm{~d}$ & 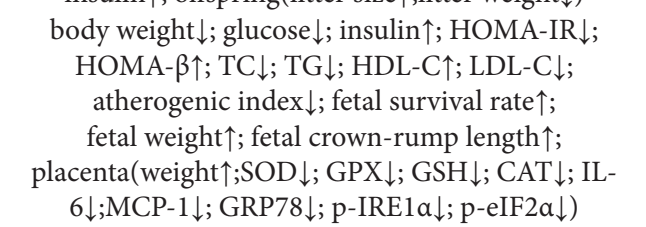 & Sha et al. (2019) \\
\hline $\mathrm{C} 57 \mathrm{BL} / \mathrm{Ks})^{\mathrm{db} /+}$ mice & Diosgenin at 10 and $20 \mathrm{mg} / \mathrm{kg}^{*} \mathrm{~d}$ & $\begin{array}{c}\text { GTT } \uparrow \text {; ITT } \uparrow \text {; glucose } \downarrow ; \text { FPG } \downarrow \text {; insulin } \downarrow \text {; hepatic } \\
\text { glycogen } \uparrow ; \text { liver } \\
\text { (TBARS } \uparrow \text {; GSH } \uparrow \text {; SOD } \uparrow \text {; CAT } \uparrow \text {; TC } \downarrow \text {; TG } \downarrow ;) \\
\text { plasma(TC } \downarrow ; \text { TG } \downarrow ; \text { LDL-C } \downarrow ; \text { HDL-C } \uparrow) ; \text { Srebp- } 1 \downarrow ; \\
\text { FAS } \downarrow ; \text { SCD- } 1 \downarrow ; \text { Acc } \downarrow\end{array}$ & Hua et al. (2016) \\
\hline $\mathrm{C} 57 \mathrm{BL} / \mathrm{Ks})^{\mathrm{db} /+}$ mice & Mogroside IIIE at $20 \mathrm{mg} / \mathrm{kg}^{*} \mathrm{~d}$ & $\begin{array}{c}\text { body weight } \downarrow \text {; glucose } \downarrow \text {; insulin } \uparrow \text {; ITT } \uparrow \text {; GTT } \uparrow \text {; birth } \\
\text { weight } \downarrow \text {; litter size } \uparrow \text {; IL- } 1 \beta \downarrow \text {; IL-6 } \downarrow \text {; TNF- } \alpha \downarrow \text {;AMPK } \\
\text { signaling } \uparrow \text {; p-AMPK/AMPK } \uparrow \text {; p-HDAC4/HDAC4 } \downarrow \text {; } \\
\text { G6P } \downarrow\end{array}$ & Zou et al. (2018) \\
\hline $\mathrm{C} 57 \mathrm{BL} / \mathrm{KsJ}{ }^{\mathrm{db} /+}$ mice & Astaxanthin at $30 \mathrm{mg} / \mathrm{kg}{ }^{*} \mathrm{~d}$ & $\begin{array}{c}\text { glucose } \downarrow ; \text { insulin } \uparrow ; \mathrm{GTT} \uparrow ; \mathrm{ITT} \uparrow ; \mathrm{TC} \downarrow ; \mathrm{TG} \downarrow ; \\
\mathrm{HDL} \uparrow ; \mathrm{LDL} \downarrow ; \mathrm{MDA} \uparrow ; \text { atherogenic index } \downarrow \text {; } \\
\text { liver(GSH } \uparrow ; \mathrm{SOD} \uparrow ; \mathrm{CAT} \uparrow ; \mathrm{MDA} \uparrow ; \mathrm{GPX} \uparrow)\end{array}$ & Chen et al. (2020) \\
\hline $\mathrm{C} 57 \mathrm{BL} / \mathrm{Ks} \int^{\mathrm{db} /+}$ mice & Apocynin at 5,20 and $50 \mathrm{mg} / \mathrm{kg}^{\star} \mathrm{d}$ & 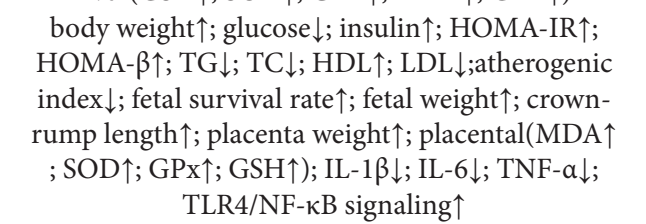 & Liu et al. (2020) \\
\hline Lepr $^{\mathrm{db}} /+(\mathrm{db} /+)$ mice & Nobiletin at $50 \mathrm{mg} / \mathrm{kg}^{*} \mathrm{~d}$ & 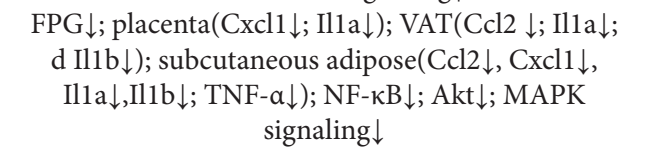 & Nguyen-Ngo et al. (2020) \\
\hline $\mathrm{C} 57 \mathrm{BL} / \mathrm{Ks} \int^{\mathrm{db} /+}$ mice & Curcumin at 50 and $100 \mathrm{mg} / \mathrm{kg}^{\star} \mathrm{d}$ & $\begin{array}{c}\text { insulin } \downarrow ; \mathrm{FPG} \downarrow ; \mathrm{GTT} \uparrow ; \mathrm{ITT} \downarrow ; \text { liver(TBARS } \downarrow \text {; GSH} \uparrow \text {; } \\
\text { SOD } \uparrow \text {; CAT } \uparrow \text { ); AMPK signaling } \uparrow \text {; p-AMPK/ } \\
\text { AMPK } \uparrow ; \mathrm{p} \text {-HDAC4/HDAC4 } \downarrow \text {; G6P } \downarrow \text {; hepatic } \\
\text { glycogen } \uparrow \text {; fetuse(birthweight } \downarrow ; \text { litter size } \uparrow)\end{array}$ & Lu et al. (2019) \\
\hline Wistar rats by STZ & $\begin{array}{l}\text { lotus leaf polysaccharides at } 50 \text { and } \\
\qquad 100 \mathrm{mg} / \mathrm{kg}^{\star} \mathrm{d}\end{array}$ & $\begin{array}{c}\text { FPG } \downarrow ; \text { FINS } \downarrow ; \text { TG } \downarrow ; \text { TC } \downarrow ; \text { HDL } \uparrow ; \mathrm{LDL} \downarrow ; \text { hepatic } \\
\text { glycogen } \uparrow ; \text { SOD } \uparrow \mathrm{CAT} \uparrow ; \mathrm{GPX} \uparrow ; \mathrm{GSH} \uparrow\end{array}$ & Wang (2013) \\
\hline Wistar rats by STZ & $\begin{array}{l}\text { lotus leaf selenium polysaccharide } \\
\text { at } 50 \text { and } 100 \mathrm{mg} / \mathrm{kg}^{*} \mathrm{~d}\end{array}$ & $\begin{array}{c}\text { FPG } \downarrow ; \text { FINS } \downarrow ; \text { TG } \downarrow ; \text { TC } \downarrow \text {; HDL } \uparrow ; \mathrm{LDL} \downarrow \text {; hepatic } \\
\text { glycogen } \uparrow \text {; liver(SOD } \uparrow ; \mathrm{GSH} \uparrow ; \mathrm{GPX} \uparrow ; \mathrm{CAT} \uparrow) \text {; fetal } \\
\text { body weight } \uparrow \text {; placental weight } \uparrow \text {; prenatal body } \\
\text { weight } \uparrow\end{array}$ & Zeng et al. (2017) \\
\hline Wistar rats by STZ & $\begin{array}{l}\text { pomegranate ellagic polyphenols at } \\
50,150 \text { and } 300 \mathrm{mg} / \mathrm{kg}^{\star} \mathrm{d}\end{array}$ & 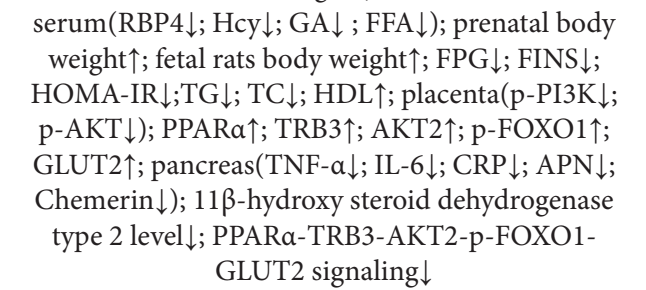 & Sun et al. (2019) \\
\hline $\begin{array}{l}\text { Sprague Dawley rats } \\
\text { by STZ }\end{array}$ & $\begin{array}{c}\text { daily } 0.5 \% \text { vitamin E plus } 1 \% \\
\text { vitamin C; daily } 2 \% \text { vitamin } \mathrm{E} \text { and } \\
4 \% \text { vitamin C }\end{array}$ & $\begin{array}{c}\text { maternal bodyweight } \uparrow \text {; maternal liverweight } \uparrow \text {; } \\
\text { glucose } \downarrow \text {; plasma(vitaminE } \uparrow \text {; vitaminC } \uparrow \text {; TBARS } \downarrow \text { ); } \\
\text { Liver(vitaminE } \uparrow \text {; vitaminC } \uparrow \text {; TBARS } \downarrow \text { ); fetal } \\
\text { livers(ascorbic acid } \uparrow \text {; a-tocopherol } \uparrow \text {; carbonylated } \\
\text { proteins to total protein amounts } \downarrow \text {; dintrophenylated } \\
\text { proteins. } \downarrow \text { ); malformations rate } \downarrow \text {; fetal weight } \uparrow \text {; fetal } \\
\text { liver weight } \uparrow\end{array}$ & Cederberg et al. (2001) \\
\hline
\end{tabular}


Table 1. Continued...

\begin{tabular}{|c|c|}
\hline Model & Intervention \\
\hline $\begin{array}{l}\text { Wistar rats (Rattus } \\
\text { norvegicus var. } \\
\text { albinus) by STZ }\end{array}$ & Lentinus edodes at $100 \mathrm{mg} / \mathrm{kg}^{\star} \mathrm{d}$ \\
\hline Albino rats by STZ & Cinnamal dehyde at $20 \mathrm{mg} / \mathrm{kg}^{\star} \mathrm{d}$ \\
\hline $\begin{array}{l}\text { Albino Wistar rats } \\
\text { by STZ }\end{array}$ & $\begin{array}{c}\text { A daily } 6 \% \text { olive oil supplemented } \\
\text { diet }\end{array}$ \\
\hline $\begin{array}{l}\text { Albino Wistar rats } \\
\text { by STZ }\end{array}$ & $\begin{array}{c}\text { A daily } 6 \% \text { olive oil supplemented } \\
\text { diet }\end{array}$ \\
\hline $\begin{array}{l}\text { Albino Wistar rats } \\
\text { by STZ }\end{array}$ & $\begin{array}{l}6 \% \text { safflflower oil Supplemented } \\
\text { diet from day } 1 \text { to } 14 \text { of } \\
\text { pregnancy,then } 6 \% \text { chia oil } \\
\text { supplemented diet from day } 14 \text { to } \\
\text { term }\end{array}$ \\
\hline $\mathrm{SD}$ rats by $\mathrm{STZ}$ & okra extract at $200 \mathrm{mg} / \mathrm{kg}^{\star} \mathrm{d}$ \\
\hline
\end{tabular}

Wistar rats by STZ

Wistar rats by STZ

Wistar rats by STZ

Wistar rats by STZ

Wistar rats by STZ

Rats by Alloxan
A daily supplemented highlypure-Cellulose-enriched-diet (10\%cellulose)

A daily $60 \%$ Omega-3 polyunsaturated fatty acids supplemented diet A daily n-3 PUFAs (EPA and DHA) supplemented diet

Zuogui Wan(rhizome of adhesive Rehmannia, Rhizoma Dioscoreae, Barbary Wolfberry fruit, Cornus officinalis, China dodder, Colla Cornus Cervi, tortoise shell glue, and medicinal Cyathula root) $100 \mathrm{mg} / \mathrm{kg}^{*}$ daqueous extract of Hibiscus rosa-sinensis from day 0 to 7 of pregnancy; $200 \mathrm{mg} / \mathrm{kg}^{*} \mathrm{~d}$ from day 8 to 14 of pregnancy; 400 $\mathrm{mg} / \mathrm{kg}^{*} \mathrm{~d}$ from day 15 to term grape seed and skin extract at $4 \mathrm{~g} / \mathrm{kg}^{*} \mathrm{~d}$

\section{GTT $\uparrow$; insulin $\downarrow$; lipase; glucose $\downarrow$; red blood cells $\uparrow$; hematocrit $\downarrow$; leukocyte $\downarrow$; hemoglobin; platelet $\uparrow ; \mathrm{HDL} \uparrow ; \mathrm{LDL} \downarrow ; \mathrm{TG} \downarrow ; \mathrm{TC} \downarrow ; \mathrm{ALT} \downarrow ; \mathrm{AST} \downarrow ;$ CAT $\uparrow$; GPX $\uparrow$; GSH $\downarrow ;$ TBARS $\downarrow$; placenta(CAT $\uparrow$; GPX $\downarrow$; GSH $\downarrow ;$ TBARS $\downarrow$ ); uterus weight $\uparrow$; ovary weight $\downarrow$; placenta weight $\downarrow$; fetal survival rate $\uparrow$} body weight $\downarrow$; glucose $\downarrow$; insulin $\uparrow$; leptin $\downarrow$; hepatic glycogen $\uparrow$; fructosamine $\downarrow$; TC $\downarrow ; \mathrm{TG} \downarrow ; \mathrm{HDL}-\mathrm{C} \uparrow$; GTT $\uparrow$; ITT $\uparrow$; birth weight $\downarrow$; litter size $\uparrow$; IL- $1 \beta \downarrow$; IL-6 $\downarrow ;$ TNF- $\alpha \downarrow$

maternal rat(TG $\downarrow ; \mathrm{TC} \downarrow)$; male offspring(TG $\downarrow ;$ TC $\downarrow$; free fatty acids $\downarrow$; phospholipids $\uparrow$; PLIN2 $\downarrow ;$ PPAR $\gamma 1 \downarrow ;$ PPAR $\gamma 2 \downarrow ;$ PPAR $\delta \downarrow ;$ PPAR $\alpha \uparrow ;$ Srebp-1c $\downarrow ;$ Acc $1 \downarrow$; Fasn $\downarrow ;$ Aoo $\downarrow ;$ Cpt1-L $\downarrow ;$ Scd-1 $\downarrow$; Cpt1-L $\downarrow$; PGC-1 $\downarrow \downarrow$; SRC- $1 \downarrow$ ); female offspring(TG $\downarrow$; TC $\uparrow$; free fatty acids $\downarrow$; cholesterylesters $\downarrow$; phospholipids $\downarrow$; PLIN2 $\uparrow$; PPAR $\gamma 1 \uparrow$; PPAR $\gamma 2 \downarrow$; PPAR $\delta \downarrow ;$ PPAR $\alpha \uparrow ;$ Srebp-1c $\uparrow$; Acc $1 \downarrow ;$ Fasn $\downarrow ; A o o \downarrow ;$ Cpt1-L $\downarrow ;$ Scd- $1 \downarrow ;$ Cpt1-L $\downarrow ;$ PGC-1 $\propto \uparrow ;$ SRC- $1 \downarrow)$ maternal rat(glucose $\downarrow$; weight $\downarrow$; CTGF $\downarrow$ ); offspring(glucose $\uparrow$; weight $\downarrow$ ); placenta(mTORC1 signaling $\uparrow$; mTORC2signaling $\uparrow$; PPAR $\gamma \downarrow ; 4 \mathrm{EBP}-1 \uparrow$; lipoperoxidation $\downarrow$; TBARS $\downarrow$; CTGF $\downarrow$; MMP $2 \downarrow$ ) maternal rat(glucose $\downarrow ; \mathrm{TG} \downarrow ; \mathrm{TC} \downarrow)$; offspring(glucose $\downarrow$; TG $\downarrow$; TC $\downarrow$; body weight $\downarrow$; lipoperoxidation $\downarrow$ ); placenta (weight $\downarrow$; mTORC2 signaling $\downarrow$; mTORC1 signaling $\downarrow$; PPAR $\gamma \uparrow$ )

prenatal body weight $\uparrow$; fetal rats weight $\uparrow$; placenta weight $\uparrow ; \mathrm{HbAl} \downarrow \downarrow$; TG $\downarrow$; TC $\downarrow$; FFA $\downarrow ;$ FPG $\downarrow$; HDL $\uparrow$; LDL $\downarrow$; FINS $\downarrow$; Serum CP $\downarrow$; hepatic glycogen $\uparrow$; liver $(\mathrm{SOD} \uparrow ; \mathrm{CAT} \uparrow ; \mathrm{GPX} \uparrow ; \mathrm{GSH} \uparrow)$; pancreas $(\mathrm{SOD} \uparrow ;$ $\mathrm{CAT} \uparrow ; \mathrm{GPX} \uparrow ; \mathrm{GSH} \uparrow)$

glucose $\downarrow$; urea $\downarrow$; creatinine $\downarrow$; uric acid $\downarrow$; albumine $\downarrow$; TC $\downarrow$; TG $\downarrow ; \mathrm{HDL} \uparrow ; \mathrm{LDL} \downarrow ; \mathrm{VLDL}-\mathrm{C} \downarrow$; VLDL-TG $\downarrow$; HDL-TG $\downarrow$; LDL-TG $\downarrow$; carbonyl proteins $\downarrow$; MDA $\downarrow$; $\mathrm{CAT} \uparrow ; \mathrm{SOD} \uparrow$

pancreas of offspring (TC $\downarrow ; \mathrm{TG} \downarrow ; \mathrm{CAT} \uparrow ; \mathrm{GSH} \uparrow$;

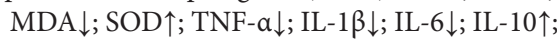
pancreatic telomere length $\uparrow$ )

offspring(body weight $\downarrow$; glucose $\downarrow$; insulin $\downarrow$; TG $\downarrow$; $\mathrm{TC} \downarrow$; adipose tissue weight $\downarrow$; adipose tissue lipid $\downarrow$; liver lipid $\downarrow)$; liver fatty acid of offspring $\left(\mathrm{C}_{20: 5 n-3} \uparrow\right.$; $\left.\mathrm{C}_{22: 6 n-3} \uparrow ; \mathrm{C}_{20: 4 n-6} \downarrow ; \mathrm{SFA} \downarrow\right)$

body weight $\downarrow ;$ FPG $\downarrow ; 2$ PPG $\downarrow$; fat weight $\downarrow$;abdominal fat weight $\downarrow$; TG $\downarrow$; TC $\downarrow$; HDL $\downarrow$; LDL $\downarrow$; insulin $\downarrow$; leptin $\downarrow ; \mathrm{APN} \uparrow ; \mathrm{HOMA}-\mathrm{IR} \downarrow$

glucose $\downarrow$; body weight gain $\uparrow$; food intake $\downarrow$; water intake $\downarrow ; \mathrm{TG} \downarrow ; \mathrm{TC} \downarrow ; \mathrm{HDL} \uparrow ; \mathrm{VLDL} \downarrow$; CRI $\downarrow$; liver(MDA $\downarrow$; GSH $\uparrow$; SOD $\uparrow$; CAT $\downarrow$ ); malformations rate $\downarrow$

body weight $\uparrow$; glycemia $\downarrow$; insulinemia $\downarrow$; prolactinemia $\downarrow$; kidney index $\downarrow$; kidney(TG $\downarrow$; G6PDH $\downarrow$; MDA $\downarrow$; protein carbonylation $\uparrow$; non protein thiols depletion $\downarrow ; \operatorname{SOD} \uparrow ; \mathrm{CAT} \uparrow) ; 1 /$ creatinine $\downarrow$; proteinuria $\downarrow$; uricemia $\downarrow$; Kidney magnesium $\uparrow$; plasma magnesium $\uparrow$; urine magnesium $\uparrow ; \mathrm{H}_{2} \mathrm{O}_{2} \downarrow ; \mathrm{AChE} \uparrow ;$ calpain $\uparrow ; \mathrm{Cu} \downarrow ; \mathrm{Se} \uparrow ;$ tyrosinase $\uparrow ; \mathrm{GPx} \uparrow$
Fornes et al. (2020)

Hosni et al. (2017)

Capobianco, et al. (2018b)

Capobianco et al. (2018a)

Tian et al. (2015)

Bensalah et al. (2018)

Gao et al. (2019a)

Soulimane-Mokhtari et al. (2005)

Wang et al. (2016)

Afiune et al. (2017)

Oueslati et al. (2016) 
Table 1. Continued...

\begin{tabular}{|c|c|c|c|}
\hline Model & Intervention & Mechanism & Reference \\
\hline Sprague Dawley rats & resveratrol at $147 \mathrm{mg} / \mathrm{kg}^{\star} \mathrm{d}$ & $\begin{array}{c}\text { energy intake } \downarrow \text {; food consumption } \downarrow ; \\
\text { FPG } \downarrow ; \text { GTT } \downarrow ; \text { ITT } \downarrow ; \text { PTT } \downarrow ; \text { FINS } \uparrow ; \text { glucagon } \uparrow ; \text { TG } \downarrow ; \\
\text { hepatic TG } \downarrow ; \text { Srebplc } \downarrow ; \text { PPAR } \alpha \uparrow ; \text { lpl } \uparrow ; \text { g6p } \uparrow ; \text { pck } 1 \downarrow ; \\
\text { offspring(food consumption } \downarrow ; \text { litter size } \uparrow ; \text { body } \\
\text { weight } \downarrow ; \text { body length } \uparrow ; \text { TG } \downarrow ; \text { Srebplc } \downarrow ; \text { PPAR } \uparrow ; \\
\text { lpl } \uparrow ; \text { G6P } \uparrow ; \text { pck } 1 \downarrow \text { ) }\end{array}$ & Brawerman et al. (2019) \\
\hline Sprague Dawley rats & $\begin{array}{l}\text { A daily } 10 \% \text { oligofructose } \\
\text { supplemented diet }\end{array}$ & $\begin{array}{c}\text { energy intake } \downarrow \text {; offspring(GTT } \uparrow ; \text { ITT } \downarrow \text {; AST } \downarrow \text {; ALT } \uparrow \text {; } \\
\text { IL- } 1 \beta \downarrow ; \text { IL- } 6 \downarrow \text {; MCP- } 1 \downarrow \text {; body weight } \downarrow \text {; body fat } \downarrow ; \\
\text { insulin } \downarrow \text {; glucose } \downarrow \text {; body fat } \downarrow \text {; leptin } \downarrow ; \text { PPAR- } \beta \downarrow \text {; } \\
\text { FABP4 } \downarrow ; \text { PGC- } 1 \alpha \downarrow) \text {; Bifidobacterium spp } \uparrow\end{array}$ & Paul et al. (2019) \\
\hline ICR mice & $\begin{array}{l}\text { A daily } 15 \% \text { lactulose } \\
\text { supplemented diet }\end{array}$ & 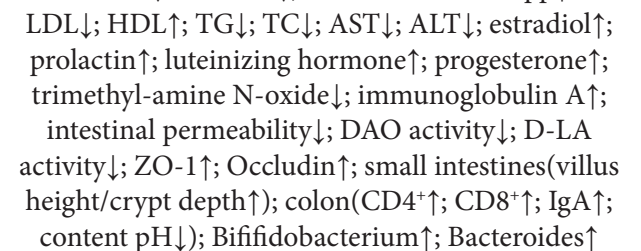 & Zhang et al. (2019) \\
\hline human & $\begin{array}{l}\text { Lycium barbarum } \mathrm{L} \text {. } \\
\text { polysaccharides at } 10 \mathrm{mg}^{\star} \mathrm{d}\end{array}$ & 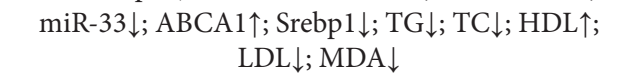 & Yang et al. (2018) \\
\hline human & $\begin{array}{l}250 \mathrm{mg}^{*} \mathrm{~d} \text { magnesium oxide plus } \\
400 \mathrm{IU}^{*} \mathrm{~d} \text { vitamin } \mathrm{E}\end{array}$ & $\begin{array}{c}\text { body weight } \downarrow ; \text { BMI } \downarrow ; \text { FPG } \downarrow \text {; insulin } \downarrow \text {; QUICKI } \uparrow ; \\
\text { HOMA-IR } \downarrow ; \text { TG } \downarrow ; \text { TC } \downarrow ; \text { LDL } \downarrow ; \text { VLDL } \downarrow\end{array}$ & Maktabi et al. (2018) \\
\hline human & soybean oligosaccharides at $10 \mathrm{~g}^{\star} \mathrm{d}$ & $\begin{array}{c}\text { HOMA-IR } \downarrow ; \text { SOD } \uparrow ; \mathrm{GPx} \uparrow ; \mathrm{CAT} \uparrow ; \mathrm{TBARS} \downarrow ; \mathrm{FPG} \downarrow ; \\
\text { FINS } \downarrow ; \mathrm{APN} \uparrow ; \mathrm{HBCI} \downarrow\end{array}$ & Fei et al. (2014) \\
\hline human & $\begin{array}{l}5 \mathrm{~g}^{\star} \mathrm{d} \text { black garlic treated } \\
\text { Lactobacillus bulgaricus }\end{array}$ & $\begin{array}{c}1 \mathrm{hPG} \downarrow ; 2 \mathrm{hPG} \downarrow ; \mathrm{FPG} \downarrow ; \mathrm{GTT} \uparrow ; \mathrm{T}-\mathrm{AOC} \uparrow ; \mathrm{SOD} \uparrow ; \\
\mathrm{GSH}-\mathrm{PX} \uparrow ; \mathrm{MDA} \downarrow\end{array}$ & Si et al. (2019) \\
\hline human & $\begin{array}{l}1000 \mathrm{mg}^{*} \mathrm{~d} \text { omega- } 3 \text { fatty acids } \\
\text { plus } 400 \mathrm{IU}^{*} \mathrm{~d} \text { vitamin } \mathrm{E}\end{array}$ & $\begin{array}{c}\text { VLDL } \downarrow ; \text { LDL } \downarrow ; \text { HDL } \uparrow ; \text { TG } \downarrow ; \text { TC } \downarrow ; \text { HOMA-IR } \downarrow ; \\
\text { HOMA- } \beta \downarrow ; \text { insulin sentivity } \downarrow\end{array}$ & Taghizadeh et al. (2016) \\
\hline human & $\begin{array}{l}\text { supplemented } 40 \mathrm{~mL} \text { extra } \\
\text { virgin olive oil plus a handful of } \\
\text { pistachios per day }\end{array}$ & $\begin{array}{c}\text { FPG } \downarrow ; \text { body weight gain } \downarrow ; \text { HOMA-IR } \downarrow \text {; } \\
\text { insulin } \downarrow ; \text { offspring(birth weight } \uparrow \text {; body length } \uparrow ; \\
\text { hypoglycemia } \downarrow \text { ) }\end{array}$ & Assaf-Balut et al. (2017) \\
\hline human & A daily high-fibres diet & VLDL $\downarrow ;$ LDL $\downarrow ;$ HDL $\uparrow ;$ GlycA $\downarrow ;$ LPS $\downarrow ;$ Lachnospira $\uparrow$ & Röytiö et al. (2018) \\
\hline human & oligosaccharide-sialic acid at $60 \mathrm{~g}^{\star} \mathrm{d}$ & $\begin{array}{c}\text { IL- } 1 \beta \downarrow ; \text { IL- } 6 \downarrow ; \text { IL- } 8 \downarrow ; \text { IL- } 10 \downarrow ; \text { TNF- } \alpha \downarrow ; \text { CRP } \downarrow ; \\
\text { VLDL } \downarrow ; \text { LDL } \downarrow ; \text { HDL } \uparrow ; \text { TG } \downarrow ; \text { TC } \downarrow ; \text { GTT } \uparrow ; \text { FPG } \downarrow\end{array}$ & Wang et al. (2019) \\
\hline human & $\begin{array}{l}\text { A daily } 2 \% \text { myo-inositol } \\
\text { supplemented diet }\end{array}$ & $\begin{array}{c}\mathrm{TG} \downarrow ; \mathrm{TC} \downarrow ; \mathrm{LDL} \downarrow ; \mathrm{HDL} \uparrow ; \mathrm{FPG} \downarrow ; \text { birth weight } \uparrow ; \\
\text { ponderal index } \downarrow\end{array}$ & Plows et al. (2020) \\
\hline human & Supplemented $2 \mathrm{~g}$ myo-inositol ${ }^{\star} \mathrm{d}$ & $\begin{array}{c}\text { Insulin } \downarrow ; \text { leptin } \uparrow ; \text { APN } \uparrow \text {; fasting c-peptide } \downarrow \text {; HOMA- } \\
\text { IR } \downarrow ; \text { GTT } \uparrow \text {; fasting glucose } \downarrow ; 2 \text { PPG } \downarrow \text {; birth weight } \downarrow \text {; } \\
\text { macrosomia rate } \downarrow\end{array}$ & Amaefule et al. (2018) \\
\hline human & $\begin{array}{l}\text { A daily Ca-rich low-fat diet } \\
\text { (200 mg Ca })\end{array}$ & $22 \%$ reduction in GDM risk & Osorio-Yáñez et al. (2017) \\
\hline human & $\begin{array}{l}\text { A daily capsule (Lactobacillus } \\
\text { acidophilus LA-5,BifidobacteriumBB- } \\
\text { 12,Streptococcus thermophilus } \\
\text { STY-31 and Lactobacillus delbrueckii } \\
\text { bulgaricus LBY-27) }\end{array}$ & body weight gain $\downarrow$; FPG $\downarrow$; FINS $\downarrow$; HOMA-IR $\downarrow$ & Dolatkhah et al. (2015) \\
\hline human & $\begin{array}{c}\text { A daily capsule (Lactobacillus } \\
\text { acidophilus, Lactobacillus casei } \\
\text { and Bififidobacterium bififidum) } \\
\text { plus } 800 \text { mg inulin }\end{array}$ & $\begin{array}{c}\text { insulin } \downarrow \text {; HOMA-IR } \downarrow \text {; HOMA-B } \downarrow ; \text { TAG } \downarrow \text {; VLDL } \downarrow ; \\
\text { TC } \downarrow ; \text { LDL } \downarrow ; \text { HDL } \downarrow\end{array}$ & Ahmadi et al. (2016) \\
\hline $\begin{array}{l}\text { in vitro human } \\
\text { placenta, adipose } \\
\text { and skeletal muscle } \\
\text { model of GDM }\end{array}$ & $\begin{array}{l}\text { Placental explants were incubated } \\
\text { in } 100 \mu \mathrm{M} \text { nobiletin;skeletal muscle } \\
\text { explants and VAT explants were } \\
\text { incubated in } 200 \mu \mathrm{m} \text { nobiletin }\end{array}$ & 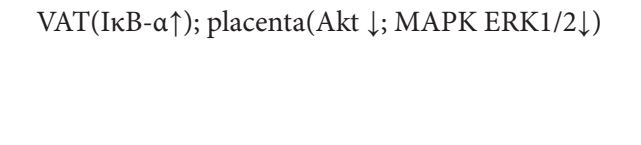 & Nguyen-Ngo et al. (2020) \\
\hline $\begin{array}{l}\text { in vitro human } \\
\text { placenta, omental } \\
\text { and subcutaneous } \\
\text { adipose tissue and } \\
\text { skeletal muscle } \\
\text { model of GDM }\end{array}$ & incubated in 200 um resveratrol & 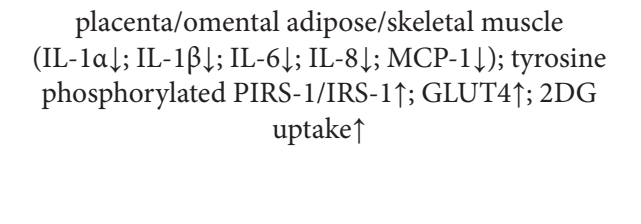 & Tran et al. (2017) \\
\hline $\begin{array}{l}\text { in vitro human } \\
\text { placental, amnion, } \\
\text { and choriodecidua } \\
\text { model of GDM }\end{array}$ & $\begin{array}{l}\text { incubated in } 50,100 \text {, and } 200 \mathrm{uM} \\
\text { resveratrol }\end{array}$ & 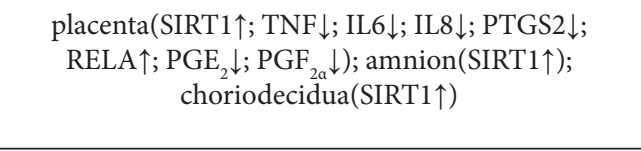 & Lappas et al. (2011) \\
\hline
\end{tabular}


attenuate GDM-induced inflammation

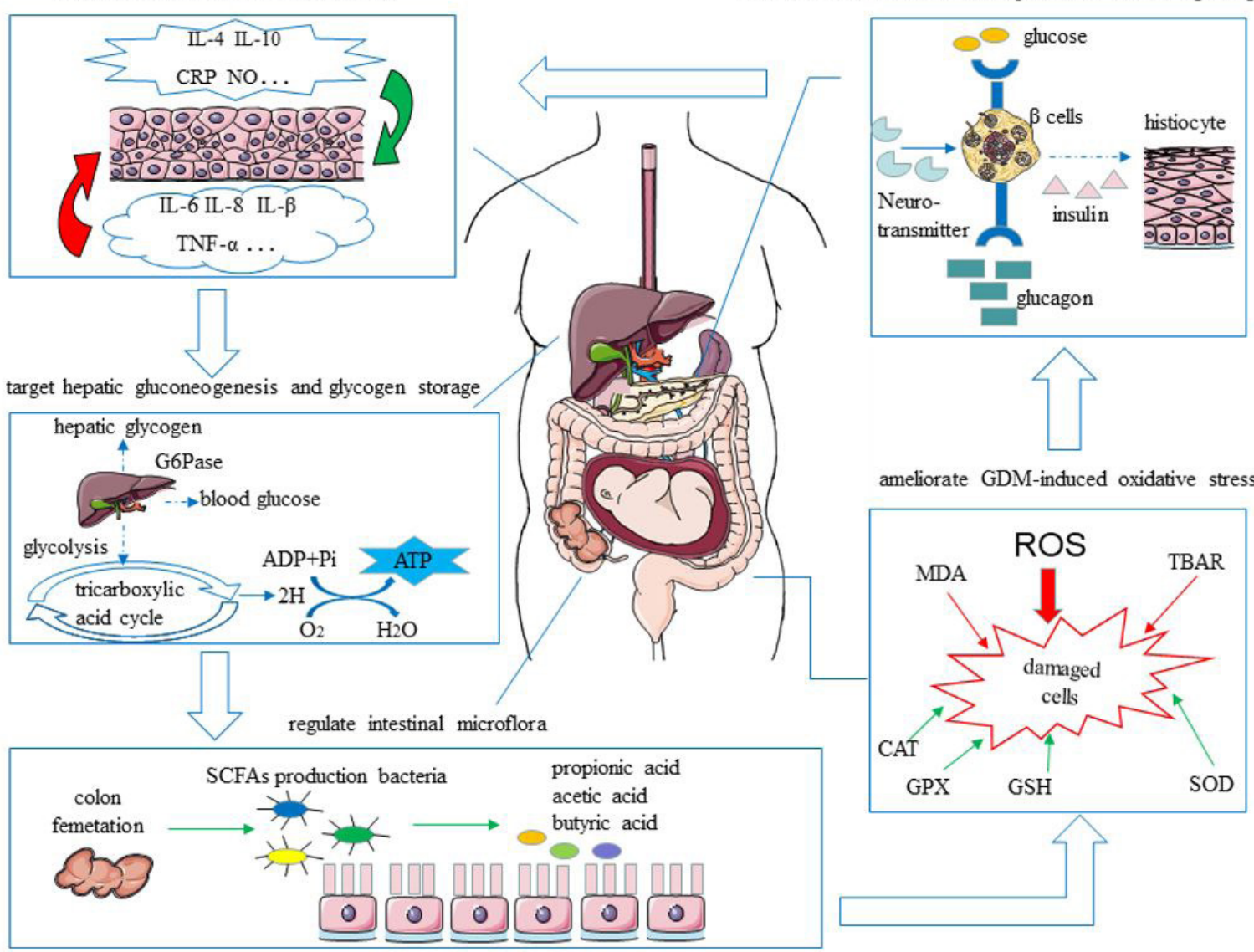

Figure 1. A schematic representation of molecular metabolism with FFIs supplementation. The metabolic pathway of insulin secretion and potentiate insulin signaling, hepatic gluconeogenesis and glycogen storage, oxidative stress, inflammation and intestinal microflora cycle overthrough the body was revealed. The red arrows indicated the negative effects, the green arrows indicated the positive effects, and the blue arrows indicated the neutral effects.

reported that a maternal diet supplemented with nobiletin inhibited mRNA expression and secretion of proinflammatory cytokines and chemokines while activating the NF- $\mathrm{kB} / \mathrm{Akt} / \mathrm{MAPK}$ signaling pathway in the placenta. Therefore, the anti-GDM effects of FFIs appear to be also mediated through inhibition of inflammatory signaling pathways.

\subsection{Correlation between the intestinal microflora and GDM}

The interplay between the intestinal microflora and energy metabolism has been studied extensively in recent years. Evidence from studies investigating changes in the intestinal microflora before and after labour revealed that women with GDM may possess unique intestinal microflora patterns significantly different to that of normal pregnancies. For instance, 1) Whole-metagenome shotgun sequencing of fecal samples revealed Parabacteroides distasonis, Klebsiella variicola, etc., were enriched in women with GDM, whereas Methanobrevibacter smithii, Alistipes spp., Bifidobacterium spp., and Eubacterium spp. were enriched in healthy controls (Kuang et al., 2017);2) OTUs assigned to Akkermansia were associated with lower insulin sensitivity, while Christensenella OTUs were associated with higher fasting plasma glucose concentrations in women with GDM (Crusell et al., 2018); 3) Ccorrelations between gut microbiome composition and circulating metabolic hormones have also been reported. For example, Gomez-Arango et al. (2016) determined that adipokine levels were strongly correlated with Ruminococcaceae and Lachnospiraceae, while insulin was positively correlated with the genus Collinsella; 4) Moreover, evidence from both rodent models and human trials support the notion that prebiotics exert significant therapeutic effects in GDM by upregulating Bifidobacterium and Bacteroides (Dolatkhah et al., 2015; Paul et al., 2019). Therefore, a range of FFIs may exert anti-GDM effects by acting similarly to prebiotics. For example, a maternal diet supplemented with lactulose increased the abundance of Bifidobacterium and Bacteroides, with significant increases in the levels of 15 metabolites (including 1-monoolein, glucose-6-phosphate, and short-chain fatty acids) and decreases in serum glucose and total cholesterol concentrations in pregnant mice (Zhang et al., 2019). Wang et al. (2019) reported that oligosaccharide-sialic acid relieved GDM by regulating the intestinal microflora, resulting in the generation of short-chain fatty acids (SCFAs). In summary, prebiotics as well as certain FFIs can exert potential therapeutic benefits for women with GDM by providing nutrient resources to specific beneficial bacteria and thus promoting a diverse and healthy intestinal microflora. In addition, prebiotics such as oligosaccharides can also be utilized as carbon sources to stimulate the production of SCFAs by gut microflora, which are 
key factors in optimising glucose homeostasis (Müller et al., 2020) and suppressing low-grade inflammation in women with GDM (Hideo et al., 2013).

\section{Conclusions}

Multiple studies support the notion that FFIs can help regulate a variety of mechanisms to ameliorate the negative outcomes associated with GDM (Figure 1 and Table 1). However, the pathogenesis of GDM remains complex, and there can be further mechanisms that are involved in the pathophysiology of GDM. Future studies are needed to further elucidate the beneficial effects and the underlying mechanisms of FFIs in the context of GDM. This may provide the basis to design the personalized dietary recommendations for women with GDM. Meanwhile, innovation in functional foods presents great opportunities to provide high-quality and affordable dietary supplements to GDM pregnancies to improve their health outcomes. For example, microencapsulation of phytochemicals can be used to protect FFIs against degradation and enhance their solubility, bioavailability (Costa et al., 2020); while development of high-fiber cookies with low glycemic index using pomace is considered as a an environment-friendly way to revalorize by-product of the juice industry (Tagliani et al., 2019; Lin et al., 2017). Notably, regarding to the GDM management with functional foods, there are many questions still to be answered. For instance, whether combining multiple FFIs can potentiate the beneficial effects of FFIs remains unknown. Relevant trials are urgently needed and should be carefully designed to account for individual differences.

In summary, considering the potential negative side effects of antidiabetic drugs (e.g., glyburide, metformin, baitangping) in women with GDM and their offspring, food supplements, in particular, FFIs could have beneficial benefits of FFIs on maternal and fetal health, without the associated side effects of drugs. We believe that in the near future, food supplements in pregnancy to combat metabolic disorders of pregnancy as GDM could become a reality.

\section{Acknowledgements}

This work was supported by China Postdoctoral Science Foundation (2019T120551, 2018M63072), Global Challenge Research Fund Visiting Fellowships; Research Fund for TaiwanStraits Postdoctoral Exchange Program (2018B003); Special Funds for Science and Technology Innovation of Fujian Agriculture and Forestry University (CXZX2019100S; CXZX2019101S); and Science Fund for Distinguished Young Scholars of Fujian Agriculture and Forestry University (xjq201908; xjq201918) for financial support.

\section{References}

Afiune, L. A. F., Leal-Silva, T., Sinzato, Y. K., Moraes-Souza, R. Q., Soares, T. S., Campos, K. E., Fujiwara, R. T., Herrera, E., Damasceno, D. C., \& Volpato, G. T. (2017). Beneficial effects of Hibiscus rosa-sinensis L. flower aqueous extract in pregnant rats with diabetes. PLoS One, 12(6), e0179785. http://dx.doi.org/10.1371/journal.pone.0179785. PMid:28644857.
Ahmadi, S., Jamilian, M., Tajabadi-Ebrahimi, M., Jafari, P., \& Asemi, Z. (2016). The effects of synbiotic supplementation on markers of insulin metabolism and lipid profiles in gestational diabetes: a randomised, double-blind, placebo-controlled trial. British Journal of Nutrition, 116(8), 1394-1401. http://dx.doi.org/10.1017/S0007114516003457. PMid:27681077.

Amaefule, C. E., Drymoussi, Z., Dodds, J., Sweeney, L., Pizzo, E., Daru, J., Robson, J., Poston, L., Khalil, A., Myers, J., Harden, A., Hitman, G. A., Khan, K., Zamora, J., Huda, M. S. B., \& Thangaratinam, S. (2018). Effectiveness and acceptability of myo-inositol nutritional supplement in the prevention of gestational diabetes (EMmY): a protocol for a randomised, placebo-controlled, double-blind pilot trial. BMJ Open, 8(9), e022831. http://dx.doi.org/10.1136/ bmjopen-2018-022831. PMid:30249632.

Assaf-Balut, C., García de la Torre, N., Durán, A., Fuentes, M., Bordiú, E., Del Valle, L., Familiar, C., Ortolá, A., Jiménez, I., Herraiz, M. A., Izquierdo, N., Perez, N., Torrejon, M. J., Ortega, M. I., Illana, F. J., Runkle, I., de Miguel, M. P., Montañez, C., Barabash, A., Cuesta, M., Rubio, M. A., \& Calle-Pascual, A. L. (2017). A Mediterranean diet with additional extra virgin olive oil and pistachios reduces the incidence of gestational diabetes mellitus (GDM): a randomized controlled trial: the St. Carlos GDM prevention study. PLoS One, 12(10), e0185873. http://dx.doi.org/10.1371/journal.pone.0185873. PMid:29049303.

Bensalah, M., Bouanane, S., Benyagoub, O., Merzouk, H., \& Fatima, Z. (2018). Effects of highly-pure-enriched-cellulose diet on digestibility, lipid metabolism and redox status in obese offspring of streptozotocin-induced diabetic rats. Experimental And Clinical Endocrinology \& Diabetes: Official Journal. German Society Of Endocrinology And German Diabetes Association, 126(2), 102-112. http://dx.doi.org/10.1055/s-0043-110014.

Brawerman, G. M., Kereliuk, S. M., Brar, N., Cole, L. K., Seshadri, N., Pereira, T. J., Xiang, B., Hunt, K. L., Fonseca, M. A., Hatch, G. M., Doucette, C. A., \& Dolinsky, V. W. (2019). Maternal resveratrol administration protects against gestational diabetes-induced glucose intolerance and islet dysfunction in the rat offspring. The Journal of Physiology, 597(16), 4175-4192. http://dx.doi.org/10.1113/JP278082. PMid:31240717.

Capobianco, E., Fornes, D., Roberti, S. L., Powell, T. L., Jansson, T., \& Jawerbaum, A. (2018a). Supplementation with polyunsaturated fatty acids in pregnant rats with mild diabetes normalizes placental PPAR $\gamma$ and mTOR signaling in female offspring developing gestational diabetes. The Journal of Nutritional Biochemistry, 53, 39-47. http:// dx.doi.org/10.1016/j.jnutbio.2017.10.006. PMid:29190548.

Capobianco, E., Gomez Ribot, D., Fornes, D., Powell, T. L., Levieux, C., Jansson, T., \& Jawerbaum, A. (2018b). Diet enriched with olive oil attenuates placental dysfunction in rats with gestational diabetes induced by intrauterine programming. Molecular Nutrition \& Food Research, 62(19), 1800263. http://dx.doi.org/10.1002/mnfr.201800263. PMid:29939470.

Cederberg, J., Simán, C. M., \& Eriksson, U. J. (2001). Combined Treatment with Vitamin E and Vitamin C Decreases Oxidative Stress and Improves Fetal Outcome in Experimental Diabetic Pregnancy. Pediatric Research, 49(6), 755-762. http://dx.doi.org/10.1203/00006450200106000-00007. PMid:11385134.

Chen, Y., Tang, J., Zhang, Y., Du, J., Wang, Y., Yu, H., \& He, Y. (2020). Astaxanthin alleviates gestational diabetes mellitus in mice through suppression of oxidative stress. Naunyn-Schmiedeberg's Archives of Pharmacology, 393(12), 2517-2527. http://dx.doi.org/10.1007/ s00210-020-01861-x. PMid:32279084.

Cho, G. J., Park, J. H., Lee, H., Yoo, S., Shin, S. A., \& Oh, M. J. (2016). Prepregnancy factors as determinants of the development of diabetes 
mellitus after first pregnancy. The Journal of Clinical Endocrinology and Metabolism, 101(7), 2923-2930. http://dx.doi.org/10.1210/ jc.2015-3761. PMid:27159192.

Costa, R. S. D., Santos, O. V., Rodrigues, A. M. C., Ribeiro-Costa, R. M., Converti, A., \& Silva, J. O. C. Jr. (2020). Functional product enriched with the microencapsulated extract of cupuassu (Theobroma grandiflorum Schum.) seed by-product. Food Science and Technology, 40(3), 543-550. http://dx.doi.org/10.1590/fst.11319.

Crusell, M. K. W., Hansen, T. H., Nielsen, T., Allin, K. H., Rühlemann, M. C., Damm, P., Vestergaard, H., Rørbye, C., Jørgensen, N. R., Christiansen, O. B., Heinsen, F. A., Franke, A., Hansen, T., Lauenborg, J., \& Pedersen, O. (2018). Gestational diabetes is associated with change in the gut microbiota composition in third trimester of pregnancy and postpartum. Microbiome, 6(1), 89. http://dx.doi. org/10.1186/s40168-018-0472-x. PMid:29764499.

Dean, S. V., Lassi, Z. S., Imam, A. M., \& Bhutta, Z. A. (2014). Preconception care: nutritional risks and interventions. Reproductive Health, 11(3, Suppl. 3), S3. http://dx.doi.org/10.1186/1742-4755-11-S3-S3. PMid:25415364.

Dolatkhah, N., Hajifaraji, M., Abbasalizadeh, F., Aghamohammadzadeh, N., Mehrabi, Y., \& Mesgari Abbasi, M. M. (2015). Is there a value for probiotic supplements in gestational diabetes mellitus? A randomized clinical trial. Journal of Health, Population and Nutrition, 33(1), 2533. http://dx.doi.org/10.1186/s41043-015-0034-9. PMid:26825666.

Farrar, D., Duley, L., Dowswell, T., \& Lawlor, D. A. (2017). Different strategies for diagnosing gestational diabetes to improve maternal and infant health. Cochrane Database of Systematic Reviews, 8(8), CD007122. http://dx.doi.org/10.1002/14651858.CD007122.pub4. PMid:28832911.

Fei, B., Ling, L., Hua, C., \& Ren, S.-y. (2014). Effects of soybean oligosaccharides on antioxidant enzyme activities and insulin resistance in pregnant women with gestational diabetes mellitus. Food Chemistry, 158, 429-432. http://dx.doi.org/10.1016/j.foodchem.2014.02.106. PMid:24731365.

Folharini, F., Orlandi, C. R., Martini, M. C., Bruxel, F., Altmayer, T., Brietzke, D. T., Gonçalves, T. E., Finatto, J., Ethur, E. M., Moura, N. F., Hoehne, L., \& Freitas, E. M. (2019). Nutritional characterization of Vasconcellea quercifolia A.St-Hil.: potential for the development of functional food. Food Science and Technology), 39(Suppl. 2), 432438. http://dx.doi.org/10.1590/fst.18018.

Fornes, D., Gomez Ribot, D., Heinecke, F., Roberti, S. L., Capobianco, E., \& Jawerbaum, A. (2020). Maternal diets enriched in olive oil regulate lipid metabolism and levels of PPARs and their coactivators in the fetal liver in a rat model of gestational diabetes mellitus. The Journal of Nutritional Biochemistry, 78, 108334. http://dx.doi.org/10.1016/j. jnutbio.2019.108334. PMid:32004928.

Gao, J., Huang, T., Li, J., Guo, X., Xiao, H., Gu, J., Tang, J., Cai, W., \& Li, D. (2019a). Beneficial effects of n-3 polyunsaturated fatty acids on offspring's pancreas of gestational diabetes rats. Journal of Agricultural and Food Chemistry, 67(48), 13269-13281. http:// dx.doi.org/10.1021/acs.jafc.9b05739. PMid:31725275.

Gao, J., Xiao, H., Li, J., Guo, X., Cai, W., \& Li, D. (2019b). N-3 polyunsaturated fatty acids decrease long-term diabetic risk of offspring of gestational diabetes rats by postponing shortening of hepatic telomeres and modulating liver metabolism. Nutrients, 11(7), 1699-1716. http://dx.doi.org/10.3390/nu11071699. PMid:31340612.

Gomez-Arango, L. F., Barrett, H. L., McIntyre, H. D., Callaway, L. K., Morrison, M., \& Dekker Nitert, M. (2016). Connections between the gut microbiome and metabolic hormones in early pregnancy in overweight and obese women. Diabetes, 65(8), 2214-2223. http:// dx.doi.org/10.2337/db16-0278. PMid:27217482.
Granato, D., Barba, F. J., Bursać Kovačević, D., Lorenzo, J. M., Cruz, A. G., \& Putnik, P. (2020). Functional foods: product development, technological trends, efficacy testing, and safety. Annual Review of Food Science and Technology, 11(1), 93-118. http://dx.doi.org/10.1146/ annurev-food-032519-051708. PMid:31905019.

Hideo, O., Yoshio, F., Chikae, K., Rie, M., Michiko, A. I., Katsumi, A., Yoshihiro, I., Shin, N., Masaru, Y., Makoto, U., \& Masamichi, I. (2013). Butyrate attenuates inflammation and lipolysis generated by the interaction of adipocytes and macrophages. Journal of Atherosclerosis \& Thrombosis, 20(5),425-442. https:// dx.doi.org/10.5551/jat.15065. PMid: 23470566

Hosni, A. A., Abdel-Moneim, A. A., Abdel-Reheim, E. S., Mohamed, S. M., \& Helmy, H. (2017). Cinnamaldehyde potentially attenuates gestational hyperglycemia in rats through modulation of PPAR $\gamma$, proinflammatory cytokines and oxidative stress. Biomedicine and Pharmacotherapy, 88, 52-60. http://dx.doi.org/10.1016/j. biopha.2017.01.054. PMid:28092845.

Hua, S., Li, Y., Su, L., \& Liu, X. (2016). Diosgenin ameliorates gestational diabetes through inhibition of sterol regulatory element-binding protein-1. Biomedicine and Pharmacotherapy, 84, 1460-1465. http:// dx.doi.org/10.1016/j.biopha.2016.10.049. PMid:27810341.

Johns, E. C., Denison, F. C., Norman, J. E., \& Reynolds, R. M. (2018). Gestational diabetes mellitus: mechanisms, treatment, and complications. Trends in Endocrinology and Metabolism, 29(11), 743-754. http:// dx.doi.org/10.1016/j.tem.2018.09.004. PMid:30297319.

Kiss, A. C., Lima, P. H., Sinzato, Y. K., Takaku, M., Takeno, M. A., Rudge, M. V., \& Damasceno, D. C. (2009). Animal models for clinical and gestational diabetes: maternal and fetal outcomes. Diabetology \& Metabolic Syndrome, 1(1), 21. http://dx.doi.org/10.1186/1758-59961-21. PMid:19840387.

Konstantinidi, M., \& Koutelidakis, A. E. (2019). Functional foods and bioactive compounds: a review of its possible role on weight management and obesity's metabolic consequences. Medicines (Basel, Switzerland), 6(3), 94. http://dx.doi.org/10.3390/medicines6030094. PMid:31505825.

Kuang, Y. S., Lu, J. H., Li, S. H., Li, J. H., Yuan, M. Y., He, J. R., Chen, N. N., Xiao, W. Q., Shen, S. Y., Qiu, L., Wu, Y. F., Hu, C. Y., Wu, Y. Y., Li, W. D., Chen, Q. Z., Deng, H. W., Papasian, C. J., Xia, H. M., \& Qiu, X. (2017). Connections between the human gut microbiome and gestational diabetes mellitus. GigaScience, 6(8), 1-12. http://dx.doi. org/10.1093/gigascience/gix058. PMid:28873967.

Kumagai, A., Itakura, A., Koya, D., \& Kanasaki, K. (2018). AMP-Activated Protein (AMPK) in Pathophysiology of Pregnancy Complications. International Journal of Molecular Sciences, 19(10), 3076. http:// dx.doi.org/10.3390/ijms19103076. PMid:30304773.

Lappas, M., Mitton, A., Lim, R., Barker, G., Riley, C., \& Permezel, M. (2011). SIRT1 is a novel regulator of key pathways of human labor. Biology of Reproduction, 84(1), 167-178. http://dx.doi.org/10.1095/ biolreprod.110.086983. PMid:20844277.

Laurino, L. F., Viroel, F. J., Caetano, E., Spim, S., Pickler, T. B., RosaCastro, R. M., Vasconcelos, E. A., Jozala, A. F., Hataka, A., Grotto, D., \& Gerenutti, M. (2019). Lentinus edodes exposure before and after fetus implantation: materno-fetal development in rats with gestational diabetes mellitus. Nutrients, 11(11), 2720. http://dx.doi. org/10.3390/nu11112720. PMid:31717560.

Lee, S. M., Kim, B. J., Koo, J. N., Norwitz, E. R., Oh, I. H., Kim, S. M., Kim, S. Y., Kim, G. M., Kwak, S. H., Kim, W., Joo, S. K., Shin, S., Vixa, C., Park, C. W., Jun, J. K., \& Park, J. S. (2019). Nonalcoholic fatty liver disease is a risk factor for large-for-gestational-age birthweight. PLoS One, 14(8), e221400. http://dx.doi.org/10.1371/ journal.pone.0221400. PMid:31449538. 
Li, S., Zhang, Y., Sun, Y., Zhang, G., Bai, J., Guo, J., Su, X., Du, H., Cao, X., Yang, J., \& Wang, T. (2019). Naringenin improves insulin sensitivity in gestational diabetes mellitus mice through AMPK. Nutrition \& Diabetes, 9(1), 28. http://dx.doi.org/10.1038/s41387019-0095-8. PMid:31591391.

Lin, S., Chi, W., Hu, J., Pan, Q., Zheng, B., \& Zeng, S. (2017). Sensory and nutritional properties of chinese olive pomace based high fibre biscuit. Emirates Journal of Food and Agriculture, 29(7), 495-501. http://dx.doi.org/10.9755/ejfa.2016-12-1908.

Liu, T., Zheng, W., Wang, L., Wang, L., \& Zhang, Y. (2020). TLR4/NF-кB signaling pathway participates in the protective effects of apocynin on gestational diabetes mellitus induced placental oxidative stress and inflammation. Reproductive Sciences, 27(2), 722-730. http:// dx.doi.org/10.1007/s43032-019-00078-5. PMid:32046403.

Lu, X., Wu, F., Jiang, M., Sun, X., \& Tian, G. (2019). Curcumin ameliorates gestational diabetes in mice partly through activating AMPK. Pharmaceutical Biology, 57(1), 250-254. http://dx.doi.org/ 10.1080/13880209.2019.1594311. PMid:30957612.

Maktabi, M., Jamilian, M., Amirani, E., Chamani, M., \& Asemi, Z. (2018). The effects of magnesium and vitamin E co-supplementation on parameters of glucose homeostasis and lipid profiles in patients with gestational diabetes. Lipids in Health and Disease, 17(1), 163. http://dx.doi.org/10.1186/s12944-018-0814-5. PMid:30025522.

Müller, M., Hermes, G. D. A., Emanuel E, C., Holst, J. J., Zoetendal, E. G., Smidt, H., Troost, F., Schaap, F. G., Damink, S. O., Jocken, J. W. E., Lenaerts, K., Masclee, A. A. M., \& Blaak, E. E. (2020). Effect of wheat bran derived prebiotic supplementation on gastrointestinal transit, gut microbiota, and metabolic health: a randomized controlled trial in healthy adults with a slow gut transit. Gut Microbes, 12(1), 1704141. http://dx.doi.org/10.1080/19490976.2019.1704141. PMid:31983281.

Nguyen-Ngo, C., Salomon, C., Quak, S., Lai, A., Willcox, J. C., \& Lappas, M. (2020). Nobiletin exerts anti-diabetic and anti-inflammatory effects in an in vitro human model and in vivo murine model of gestational diabetes. Clinical Science, 134(6), 571-592. http://dx.doi. org/10.1042/CS20191099. PMid:32129440.

Osorio-Yáñez, C., Qiu, C., Gelaye, B., Enquobahrie, D. A., \& Williams, M. A. (2017). Risk of gestational diabetes mellitus in relation to maternal dietary calcium intake. Public Health Nutrition, 20(6), 1082-1089. http://dx.doi.org/10.1017/S1368980016002974. PMid:27964774.

Oueslati, N., Charradi, K., Bedhiafi, T., Limam, F., \& Aouani, E. (2016). Protective effect of grape seed and skin extract against diabetesinduced oxidative stress and renal dysfunction in virgin and pregnant rat. Biomedicine and Pharmacotherapy, 83, 584-592. http://dx.doi. org/10.1016/j.biopha.2016.07.024. PMid:27459114.

Pantham, P., Aye, I. L. M. H., \& Powell, T. L. (2015). Inflammation in maternal obesity and gestational diabetes mellitus. Placenta, 36(7), 709-715. http://dx.doi.org/10.1016/j.placenta.2015.04.006. PMid:25972077.

Pasek, R. C., \& Gannon, M. (2013). Advancements and challenges in generating accurate animal models of gestational diabetes mellitus. American Journal of Physiology, Endocrinology and Metabolism, 305(11), E1327-E1338. http://dx.doi.org/10.1152/ajpendo.00425.2013. PMid:24085033.

Patti, A. M., Giglio, R. V., Pafili, K., Rizzo, M., \& Papanas, N. (2018). Pharmacotherapy for gestational diabetes. Expert Opinion on Pharmacotherapy, 19(13), 1407-1414. http://dx.doi.org/10.1080/1 4656566.2018.1509955. PMid:30136869.

Paul, H. A., Collins, K. H., Nicolucci, A. C., Urbanski, S. J., Hart, D. A., Vogel, H. J., \& Reimer, R. A. (2019). Maternal prebiotic supplementation reduces fatty liver development in offspring through altered microbial and metabolomic profiles in rats. The FASEB Journal, 33(4), 51535167. http://dx.doi.org/10.1096/fj.201801551R. PMid:30629464.

Plows, J. F., Ramos Nieves, J. M., Budin, F., Mace, K., Reynolds, C. M., Vickers, M. H., Silva-Zolezzi, I., Baker, P. N., \& Stanley, J. L. (2020). The effects of myo-inositol and probiotic supplementation in a highfat-fed preclinical model of glucose intolerance in pregnancy. British Journal of Nutrition, 123(5), 516-528. http://dx.doi.org/10.1017/ S0007114519003039. PMid:31771683.

Ponzo, V., Fedele, D., Goitre, I., Leone, F., Lezo, A., Monzeglio, C., Finocchiaro, C., Ghigo, E., \& Bo, S. (2019). Diet-gut microbiota interactions and gestational diabetes mellitus (GDM). Nutrients, 11(2), 330. http://dx.doi.org/10.3390/nu11020330. PMid:30717458.

Röytiö, H., Mokkala, K., Vahlberg, T., \& Laitinen, K. (2018). Dietary intake of fat and fibre according to reference values relates to higher gut microbiota richness in overweight pregnant women CORRIGENDUM. British Journal of Nutrition, 120(5), 599-600. http://dx.doi.org/10.1017/S0007114518001940. PMid:30060769.

Sattari, M., Bril, F., Egerman, R., Kalavalapalli, S., \& Cusi, K. (2020). Relationship between non-alcoholic fatty liver disease during pregnancy and abnormal glucose metabolism during and after pregnancy. Journal of Investigative Medicine, 68(3), 743-747. http:// dx.doi.org/10.1136/jim-2019-001186. PMid:31852748.

Sha, H., Zeng, H., Zhao, J., \& Jin, H. (2019). Mangiferin ameliorates gestational diabetes mellitus-induced placental oxidative stress, inflammation and endoplasmic reticulum stress and improves fetal outcomes in mice. European Journal of Pharmacology, 859, 172522. http://dx.doi.org/10.1016/j.ejphar.2019.172522. PMid:31276667.

Si, L., Lin, R., Jia, Y., Jian, W., Yu, Q., Wang, M., \& Yang, S. (2019). Lactobacillus bulgaricus improves antioxidant capacity of black garlic in the prevention of gestational diabetes mellitus: a randomized control trial. Bioscience Reports, 39(8), BSR20182254. http://dx.doi. org/10.1042/BSR20182254. PMid:31362999.

Soulimane-Mokhtari, N. A., Guermouche, B., Yessoufou, A., Saker, M., Moutairou, K., Hichami, A., Merzouk, H., \& Khan, N. A. (2005). Modulation of lipid metabolism by $n-3$ polyunsaturated fatty acids in gestational diabetic rats and their macrosomic offspring. Clinical Science, 109(3), 287-295. http://dx.doi.org/10.1042/CS20050028. PMid:15898958.

Stewart, Z. A. (2020). Gestational diabetes. Obstetrics, Gynaecology and Reproductive Medicine, 30(3), 79-83. http://dx.doi.org/10.1016/j. ogrm.2019.12.005.

Sun, Y.-L., Zhou, F.-M., \& Wang, H.-R. (2019). Mechanism of pomegranate ellagic polyphenols reducing insulin resistance on gestational diabetes mellitus rats. American Journal of Translational Research, 11(9), 5487-5500. http://dx.doi.org/10.1113/JP278082. PMid:31632524.

Taghizadeh, M., Jamilian, M., Mazloomi, M., Sanami, M., \& Asemi, Z. (2016). A randomized-controlled clinical trial investigating the effect of omega-3 fatty acids and vitamin E co-supplementation on markers of insulin metabolism and lipid profiles in gestational diabetes. Journal of Clinical Lipidology, 10(2), 386-393. http://dx.doi. org/10.1016/j.jacl.2015.12.017. PMid:27055970.

Tagliani, C., Perez, C., Curutchet, A., Arcia, P., \& Cozzano, S. (2019). Blueberry pomace, valorization of an industry by-product source of fibre with antioxidant capacity. Food Science and Technology, 39(3), 644-651. http://dx.doi.org/10.1590/fst.00318.

Tian, Z.-H., Miao, F.-T., Zhang, X., Wang, Q.-H., Lei, N., \& Guo, L.-C. (2015). Therapeutic effect of okra extract on gestational diabetes mellitus rats induced by streptozotocin. Asian Pacific Journal of Tropical Medicine, 8(12), 1038-1042. http://dx.doi.org/10.1016/j. apjtm.2015.11.002. PMid:26706676. 
Tran, H. T., Liong, S., Lim, R., Barker, G., \& Lappas, M. (2017). Resveratrol ameliorates the chemical and microbial induction of inflammation and insulin resistance in human placenta, adipose tissue and skeletal muscle. PLoS One, 12(3), e0173373. http://dx.doi. org/10.1371/journal.pone.0173373. PMid:28278187.

Wahli, W., \& Michalik, L. (2012). PPARs at the crossroads of lipid signaling and inflammation. Trends in Endocrinology and Metabolism, 23(7), 351-363. http://dx.doi.org/10.1016/j.tem.2012.05.001. PMid:22704720.

Wang, H. (2013). Preventive effects of ophiopogon-polysaccharide on apiponectin in gestational diabetes mellitus rat. Asian Pacific Journal of Tropical Medicine, 6(4), 296-299. http://dx.doi.org/10.1016/S19957645(13)60059-0. PMid:23608331.

Wang, S., Peng, R., Qin, S., Liu, Y., Yang, H., \& Ma, J. (2019). Effects of oligosaccharide-sialic acid (OS) compound on maternal-newborn gut microbiome, glucose metabolism and systematic immunity in pregnancy: protocol for a randomised controlled study. BMJ Open, 9(9), e026583. http://dx.doi.org/10.1136/bmjopen-2018-026583. PMid:31511279.

Wang, Y., Feng, Q., Niu, X., Xu, K., Wang, Y., Wang, J., Li, Q., Mao, Y., \& Gao, S. (2016). The preventive effect of zuogui wan on offspring rats' impaired glucose tolerance whose mothers had gestational diabetes mellitus. Evidence-Based Complementary and Alternative Medicine, 2016, 9417362. http://dx.doi.org/10.1155/2016/9417362. PMid:27034700.

Xing, B.-H., Yang, F.-Z., \& Wu, X.-H. (2016). Naringenin enhances the efficacy of human embryonic stem cell-derived pancreatic endoderm in treating gestational diabetes mellitus mice. Journal of Pharmacological Sciences, 131(2), 93-100. http://dx.doi.org/10.1016/j. jphs.2016.04.014. PMid:27156928.

Yang, S., Si, L., Fan, L., Jian, W., Pei, H., \& Lin, R. (2018). Polysaccharide IV from Lycium barbarum L. improves lipid profiles of gestational diabetes mellitus of pregnancy by upregulating ABCA1 and downregulating sterol regulatory element-binding transcription 1 via miR-33. Frontiers in Endocrinology, 9, 49. http://dx.doi.org/10.3389/ fendo.2018.00049. PMid:29527188.

Yao, L., Wan, J., Li, H., Ding, J., Wang, Y., Wang, X., \& Li, M. (2015). Resveratrol relieves gestational diabetes mellitus in mice through activating AMPK. Reproductive Biology and Endocrinology, 13(1), 118125. http://dx.doi.org/10.1186/s12958-015-0114-0. PMid:26542478.

Zeng, Z., Xu, Y., \& Zhang, B. (2017). Antidiabetic activity of a lotus leaf selenium (Se)-polysaccharide in rats with gestational diabetes mellitus. Biological Trace Element Research, 176(2), 321-327. http:// dx.doi.org/10.1007/s12011-016-0829-6. PMid:27580896.

Zhang, R., Xing, B., Zhao, J., Zhang, X., Zhou, L., Yang, S., Wang, Y., \& Yang, F. (2020). Astragaloside IV relieves gestational diabetes mellitus in genetic mice through reducing hepatic gluconeogenesis. Canadian Journal of Physiology and Pharmacology, 98(7), 466-472. http://dx.doi.org/10.1139/cjpp-2019-0548. PMid:32160476.

Zhang, Z., Chen, X., Zhao, J., Tian, C., Wei, X., Li, H., Lin, W., Jiang, A., Feng, R., Yuan, J., \& Zhao, X. (2019). Effects of a lactulose-rich diet on fecal microbiome and metabolome in pregnant mice. Journal of Agricultural and Food Chemistry, 67(27), 7674-7683. http://dx.doi. org/10.1021/acs.jafc.9b01479. PMid:31132256.

Zou, C., Zhang, Q., \& Zhang, S. (2018). Mogroside IIIE attenuates gestational diabetes mellitus through activating of AMPK signaling pathway in mice. Journal of Pharmacological Sciences, 138(3), 161166. http://dx.doi.org/10.1016/j.jphs.2018.09.008. PMid:30316692.

Zygula, A., Kosinski, P., Zwierzchowska, A., Sochacka, M., Wroczynski, P., Makarewicz-Wujec, M., Pietrzak, B., Wielgos, M., Rzentala, M., \& Giebultowicz, J. (2019). Oxidative stress markers in saliva and plasma differ between diet-controlled and insulin-controlled gestational diabetes mellitus. Diabetes Research and Clinical Practice, 148, 72-80. http://dx.doi.org/10.1016/j.diabres.2018.11.021. PMid:30529575. 